\title{
Non-linear viscoelastic response of magnetic fiber suspensions in oscillatory shear
}

\author{
P. Kuzhir ${ }^{\text {a* }}$, A. Gómez-Ramírez ${ }^{\text {b }}$, M.T. López-López ${ }^{\text {, }}$ G. Bossis ${ }^{\mathrm{a}}$, A. Yu. Zubarev ${ }^{\mathrm{c}}$ \\ ${ }^{\text {a }}$ Laboratory of Condensed Matter Physics, University of Nice - Sophia Antipolis, Parc Valrose 06108 Nice \\ Cedex 2 France, kuzhir@unice.fr, bossis@unice.fr \\ ${ }^{\mathrm{b}}$ Department of Applied Physics, University of Granada, Avda. Fuentenueva s/n, 18017, Granada, Spain, \\ anagr@ugr.es, modesto@ugr.es \\ ${ }^{\mathrm{c}}$ Department of Mathematical Physics, Ural State Univeristy, Lenin Av. 51, 620083 Ekaterinburg, Russia, \\ Andrey.Zubarev@usu.ru \\ * Corresponding author: e-mail: kuzhir@ unice.fr, tel: +33492076716, fax: +33492076536
}

\begin{abstract}
This paper reports the first study on the large amplitude oscillatory shear flow for magnetic fiber suspensions subject to a magnetic field perpendicular to the flow. The suspensions used in our experiments consisted of cobalt microfibers of the average length of $37 \mu \mathrm{m}$ and diameter of $4.9 \mu \mathrm{m}$, dispersed in a silicon oil. Rheological measurements have been carried out at imposed stress using a controlled stress magnetorheometer. The stress dependence of the shear moduli presented a staircase-like decrease with, at least, two viscoelastic quasiplateaus corresponding to the onset of microscopic- and macroscopic scale rearrangement of the suspension structure, respectively. The frequency behavior of the shear moduli followed a power-law trend at low frequencies and the storage modulus showed a high-frequency plateau, typical for Maxwell behavior. Our simple single relaxation time model fitted reasonably well the rheological data. To explain a relatively high viscous response of the fiber suspension, we supposed a coexistence of percolating and pivoting aggregates. Our simulations revealed that the former became unstable beyond some critical stress and broke in their middle part. At high stresses, the free aggregates were progressively destroyed by shear forces that contributed to a drastic decrease of the moduli. We have also measured and predicted the output strain waveforms and stress-strain hysteresis loops. With the growing stress, the shape of the stress-strain loops changed progressively from near-ellipsoidal one to the rounded-end rectangular one due to a progressive transition from a linear viscoelastic to a viscoplastic Bingham-like behavior.
\end{abstract}

Keywords: magnetorheology; fiber suspension; non-linear viscoelasticity; oscillatory shear

\section{Introduction}

Magnetorheological (MR) fluids are suspensions of magnetic micron-sized particles in a carrier liquid. The MR fluid particles are subject to a reversible aggregation and the MR fluid develops a large yield stress upon application of a magnetic field. This phenomenon is known as the magnetorheological effect $[1,2]$. So, the magnetic and mechanical properties of these fluids can be efficiently controlled by external magnetic fields and this makes them attractive for several industrial applications. Controlled stiffness dampers and magnetorheological 
finishing of optical surfaces have been successfully commercialized during the last decade and the other magnetorheological smart devices, such as controlled hydrodynamic bearings, are being developed [3]. The improvement of the real time control of these devices and the increase of the controllability range of their control parameters (flow rate, stiffness) are still important issues. In general, there are two ways of solving these problems: either by optimization of the fluidic and the magnetic designs of the considered device or by improving the properties of the MR fluid.

One way of improvement of the MR fluid stability and mechanical properties consists of using magnetic micro- or nanofibers instead of conventional spherical particles. The magnetic fiber suspensions have shown better sedimentation stability [4] and developed a yield stress a few times larger than that of the magnetic suspensions of spherical particles at the same magnetic field intensities and the same particle volume fraction [5-10]. Such enhanced magnetorheological effect in fiber suspensions have been explained in terms of the interfiber solid friction [11] and by enhanced magnetic permeability of these suspensions as compared to the permeability of conventional MR fluids $[9,12]$. Note that the similar particle shape effect has been observed in electrorheological (ER) fluids [13-16] and was attributed to both the physical overlapping of the elongated particles and to their strong dielectric properties [17-18]. Kor and See [19] have recently carried out particle level simulations on ER fluids and also found the stress enhancement in suspensions of needle-like particles.

Almost all the studies on elongated particle-based MR or ER fluids were focused on the steady shear or the small amplitude oscillatory shear flows. These studies give an important insight into the understanding of the general rheological behavior of the suspensions but have limited applications in ER and MR smart devices. For instance, in MR dampers, the MR fluid is subject to a reciprocal large amplitude straining motion. Thus, investigation of the large amplitude oscillatory shear (LAOS) flow of magnetic fiber suspensions would be very helpful for this application. Apart from its engineering importance, the LAOS response of the magnetic fiber suspensions could bring valuable information on their nonlinear viscoelasticity in the wide range of the excitation frequencies.

In general, the LAOS tests have been successfully used to characterize the rheological properties of various kinds of soft materials, such as polymers, wormlike micelles, colloidal gels, biopolymer networks, see for instance [20, 21]. These tests consist of imposing a sinusoidal strain (or stress) to the material and recording its stress (or, respectively, strain) response. In the nonlinear viscoelastic regime, i.e. at large strains or stresses applied, the material response is non-sinusoidal with higher harmonics appearing. Fourier analysis of the measured response signal allows one to learn about a rheological behavior exhibited by the material (shear thinning/thickening, strain hardering/softenning). The general framework of treatment and interpretation of the LAOS data has been developed by Wilhelm [22] and Ewoldt et al. [23].

A number of works has been devoted to the LAOS response of conventional ER fluids and MR fluids, both composed of spherical particles [24-32]. The electrorheological and 
magnetorheological effects are essentially similar in nature, so both ER and MR fluids show similar behavior in oscillatory shear. In most of the works, the first harmonic (or fundamental) shear moduli were used as the principle measure of the viscoelastic response of these fluids. In experiments, both storage and loss moduli have been found to increase with the magnetic (or electric) field strength because of the field-induced aggregation of the MR (or ER) fluids. At small strains, the aggregates spanned the gap between rheometer plates and the MR and ER fluids showed an elastic behavior with the storage modulus substantially larger than the loss modulus. Nevertheless, the loss modulus was non negligible and even a few orders of magnitude larger than the one of the dispersing liquid of the suspension. Such viscous response of the suspensions was interpreted in the two following ways. First, McLeish et al. [33] supposed a co-existence in the ER fluid of the gap-spanning particle chains with the free chains attached to the wall by one of the ends. The gap-spanning chains were supposed to move affinely with the suspending liquid and contributed to the suspension storage modulus, while the free chains moved out of phase with the rheometer walls and bended under the hydrodynamic forces exerted by the suspending liquid. Thus, a small fraction of the free chains contributed to the suspension loss modulus. Moreover, different chain bending modes gave an infinite series of relaxation times. To fit their theory to experimental data on the loss modulus, the authors adjusted the volume fraction of the free chains. On the other hand, Klingenberg [34] has carried out particle level simulations and shown that the non negligible viscous response of the ER fluids could arise from non-affine motion of the particles inside the gap-spanning aggregates even at strains as small as $10^{-4}$.

With increasing strain amplitude, the storage modulus experiences a slow decrease until some critical strain followed by an abrupt decrease above this strain. The loss modulus also decreases with the strain but in a less extent, so that, at large strains, both moduli are usually of the same order of magnitude signifying the transition from solid to liquid like behavior [35]. Parthasarathy and Klingenberg [26, 27] explained the smooth initial decrease of the storage modulus by a short-scale rearrangement of particles in aggregates and they interpreted the further abrupt drop of the modulus by the large-scale structure rearrangement and by rupture and reformation of gap-spanning structures.

In most cases, the frequency dependence of the shear moduli of ER and MR fluids followed a Maxwell-like behavior with the storage modulus increasing monotonically and attaining a high frequency plateau and the loss modulus having a maximum at intermediate frequency. Such dependence supports, at least qualitatively, both Mc Leish's "free chain hypothesis" [33] and Parthasarathy and Klingenberg's [27] observations of small and large scale cluster rearrangements.

A deeper understanding of the nonlinear viscoelastic response of the ER and MR fluid could be achieved by analyzing the stress-strain hysteresis loops, called Lissajous plots. When increasing the amplitude of the applied strain, the suspension stress response becomes nonlinear and the Lissajous plots change their shape from the ellipsoidal shape at low strains to the parallelogram-like one at high strains [24]. Martin and Odinek [25] have attributed this particular shape of the Lissajous plots to rapid fragmentation and aggregation of particle 
chains in oscillatory shear. They have proposed a phenomenological equation describing kinetics of chain aggregation/fragmentation and developed a theoretical model, which reproduced the experimental hysteresis loops reasonably well with a single fit parameter. Parthasarathy and Klingenberg [27] have also well reproduced the parallelogram-like hysteresis loops in their simulations and explained them in terms of plastic Bingham-like behavior. Namely, as the direction of shear is reversed, the stress varies rapidly until the structure yields, and the stress becomes independent of strain.

Tracking back to the ER and MR fluids composed of elongated particles, only the paper of Tsuda et al. [15] reports on the nonlinear viscoelastic response of such fluids - the ER whisker suspension. In their paper, the authors measured the complex shear modulus as function of the applied stress and have found a similar abrupt decrease of the modulus with increasing stress as in the case of suspensions of spherical particles. However, the solid-toliquid transition in whisker suspensions (corresponding to the drop of the modulus) occurred at higher critical stresses. This was explained by the formation of branched intricate structures in whisker suspensions that were mechanically stiffer than the column structures in suspensions of spheres. No results have been presented on the stress and strain waveforms. Theoretical models have neither been reported to describe the LAOS response of the ER or MR suspensions of elongated particles. It is worth to mention the dipole interaction models of Kanu and Shaw [17] and de Vicente et al. [9] that predicted the elastic modulus of, respectively, ER and MR suspensions of needle-like particles in the linear viscoelastic regime at small applied stresses or strains.

In this paper we have carried out a detailed investigation of the large amplitude oscillatory shear flow of the magnetic fiber suspensions in the presence of an external magnetic field. In experiments, we apply a sinusoidal shear stress and measure the output strain waveform. We examine effects of the magnetic field strength, stress amplitude and excitation frequency on the strain signal as well as on the shear moduli of the fiber suspension. Particular attention is focused on the stress dependence of the loss modulus which, to our opinion, has never been clearly explained even for conventional suspensions of spherical particles. Finally, we develop a microstructural model that allows describing, at least semi-quantitatively, all observed rheological behaviors in the non-linear viscoelastic regime. In particular, to explain relatively high values of the loss modulus, we assume a specific structure of the fiber suspension, composed of both percolating aggregates and the free branches attached by one extremity to either the walls of a flow cell or to the percolating aggregates. These free branches move out of phase with the suspending liquid and therefore contribute to the loss modulus. Having some similarities with McLeish's string model [33], the one reported in the present paper seems to be more realistic and more appropriate to numerous branched structures that exist in fiber suspensions.

This paper is organized as follows. In the next Section 2, we present experimental tools and methods used in this study. In the Section 3, we develop a theoretical model allowing us to predict the shear moduli and the output strain waveforms in the regime of the applied sinusoidal stress. Both experimental and theoretical results are reported and compared in the 
$4^{\text {th }}$ Section. In the last Section 5, the main conclusions are outlined and some perspectives are proposed for the further investigations.

\section{Experimental}

The magnetic fiber suspension used in our study was composed of rod-like cobalt particles dispersed in the silicon oil (Rhodorsil @; VWR International, dynamic viscosity at $25^{\circ} \mathrm{C}$ is $0.485 \mathrm{~Pa} \cdot \mathrm{s}$ ) at the volume fraction $\Phi=5 \%$ and stabilized by an appropriate amount of aluminum stearate (Sigma-Aldrich). The cobalt particles were synthesized via a polyol method described in [7] and were $37 \pm 3 \mu \mathrm{m}$ in length and $4.9 \pm 1.0 \mu \mathrm{m}$ in diameter. The magnetization curve of these particles followed the Fröhlich-Kennely law [36] with the saturation magnetization $M_{S}=1366 \pm 8 \mathrm{kA} / \mathrm{m}$ [7] and the initial magnetic susceptibility $\chi_{i} \approx 70$. The dispersion of the particles in the silicon oil was performed according to the procedure described in $[7,8]$.

The oscillatory shear tests were carried out using the controlled-stress rheometer Thermo Haake RS150 equipped with the software allowing recording of the stress and strain waveforms. The titanium plate-plate geometry was used with the disk diameter of $35 \mathrm{~mm}$ and the gap between plates equal to $0.2 \mathrm{~mm}$. A homogeneous magnetic field perpendicular to the rheometer plates was created by a solenoid placed around the measuring geometry.

The experimental protocol was as follows. At the beginning of each measurement, the suspension was presheared at $50 \mathrm{~s}^{-1}$ during $1 \mathrm{~min}$. Then, the magnetic field was applied and the suspension was kept at rest during $1 \mathrm{~min}$. After that, we proceeded to either stress sweep or frequency sweep measurement. In both measurements, a sinusoidal stress of a fixed amplitude and frequency was applied to the suspension during the time necessary to produce 20 periods of oscillations. The strain waveforms were recorded during the last 5 periods. Then, one of the parameters (stress amplitude or excitation frequency) increased in a step manner, the other parameter kept fixed, and the corresponding waveforms were recorded. In this manner, we realized the stress ramp from $\sigma_{0}=0.4 \mathrm{~Pa}$ to $400 \mathrm{~Pa}$ at the frequency $f=1 \mathrm{~Hz}$ and the frequency ramp from $f=0.05 \mathrm{~Hz}$ to $50 \mathrm{~Hz}$ at two stress amplitudes, $\sigma_{0}=30 \mathrm{~Pa}$ and $244 \mathrm{~Pa}$. The stress sweep was done for six values of the applied external magnetic field: 0, 6.1, 12.2, 18.3, 20.4 and $30.6 \mathrm{kA} / \mathrm{m}$, while the frequency sweep was done for the strongest magnetic field, $H_{0}=30.6 \mathrm{kA} / \mathrm{m}$. We estimated inertia torques generated by the rheometer at high-frequency limit and excluded those experimental points, for which the ratio of inertia torque to the applied (viscoelastic) torque was larger than $25 \%$. For each test, the experimental waveforms and Lissajous stress-strain loops were plotted and analyzed. In addition to it, the first harmonic storage and loss moduli were plotted as function of either applied stress or excitation frequency.

\section{Theory}

In this section, we develop a microstructural model of the magnetic fiber suspension under oscillatory shear allowing us to relate the structure parameters (aggregates' orientation and size) to the macroscopic rheological properties of the suspension. Consider a fiber suspension 
sandwiched between two parallel plates and subject to an external magnetic field, $\mathbf{H}_{0}$, perpendicular to the plates, as shown in Fig.1a. The lower plate is at rest and the upper one performs a reciprocal motion with the velocity $\mathbf{v}$, and involves the suspension into oscillatory flow with a presumably linear velocity profile, $v(t)=\dot{\gamma}(t) z$, where $\dot{\gamma}(t)$ is the time-dependent shear rate. Oscillatory motion of the suspension is induced by an applied sinusoidal stress $\sigma(t)=\sigma_{0} \cos \omega t$, where $\omega=2 \pi f$ is the angular frequency of oscillations, $f$ is the excitation frequency, $\sigma_{0}$ is the stress amplitude.

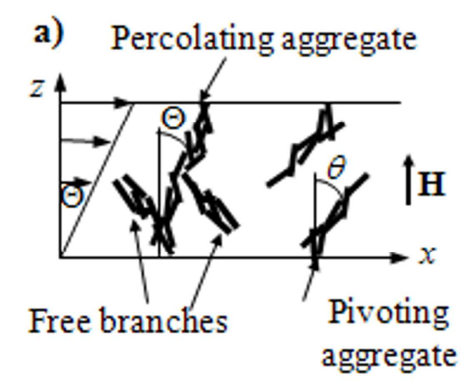

c) $\sigma<\sigma_{1}$

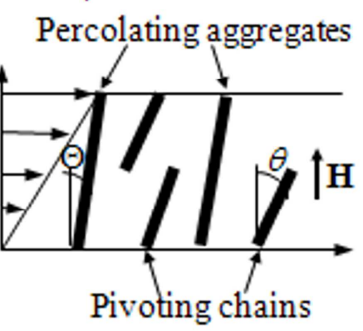

f) $\sigma_{3}<\sigma<\sigma_{4}$

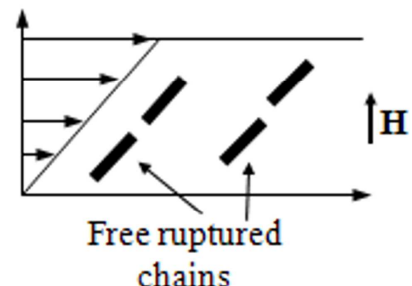

d) $\sigma_{1}<\sigma<\sigma_{2}$

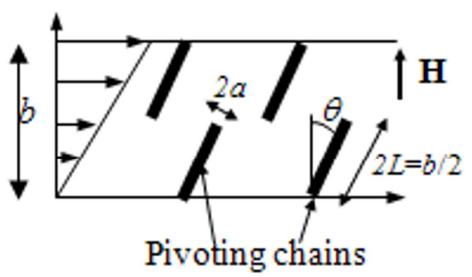

g) $\sigma>\sigma_{4}$

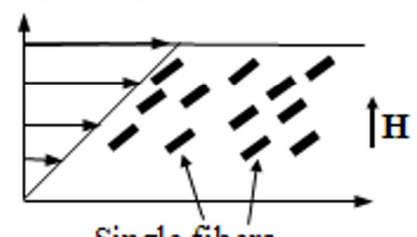

Single fibers b)

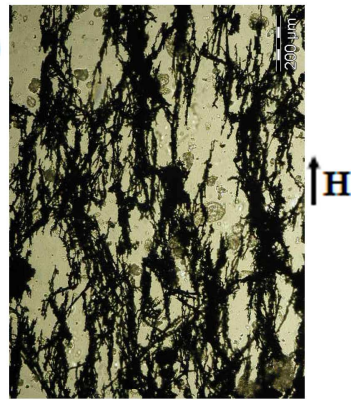

e) $\sigma_{2}<\sigma<\sigma_{3}$

Free unruptured

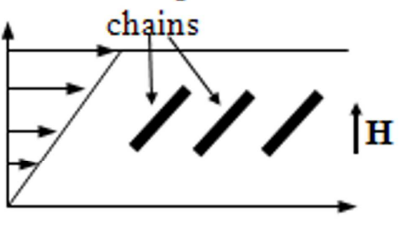

h)

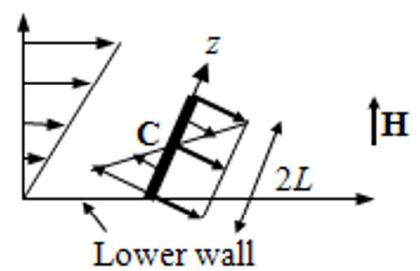

Fig.1. Microstructure of the magnetic fiber suspension: (a) - realistic representation of the suspension structure with percolating and pivoting aggregates and free branches of aggregates, all of them composed of non perfectly aligned fibers; (b) - the optical microscopy picture of the structure of quiescent fiber suspension subject to a magnetic field, $H_{0}=25 \mathrm{kA} / \mathrm{m}$ [8]. In figures (c) $-(\mathrm{g})$ the schematic representation of different microstructures evolving with increasing oscillation amplitude is shown: (c) - coexisting percolating aggregates and pivoting chains; (d) - pivoting chains; (e) - free chains detached from the walls; (f) - free chains ruptured by the tensile hydrodynamic force; (g) -single non-aggregated fibers at high stresses applied. In figure (h), a sketch for the calculation of the hydrodynamic torque on a pivoting chain is presented; in the coordinate frame of the chain, the solvent velocity profile is decomposed into a constant velocity profile at the chain center C (bold arrows) and a linear velocity profile (thin arrows).

The following assumptions and considerations are used in our model:

1. According to optical observations of the quiescent magnetic fiber suspensions performed in our previous work [8], in the presence of the magnetic field, the fibers are attracted to each 
other and form a non-regular intricate network, as shown in Fig.1b. In such network, one can distinguish percolating aggregates extending between two extremities of the cell and free branches of different sizes, attached by one end to the percolating aggregates and having another end free. We suppose the existence of these types of aggregates in oscillatory shear flow, at least at small-to-moderate amplitudes. In particular, the suspension consists of a number of percolating aggregates, some non-percolating aggregates attached by one of the ends to the upper or the lower plate and some free branches attached by one of the ends to percolating aggregates, as depicted schematically in Fig. 1a. The motion of percolating aggregates is affine so that they contribute only to the elastic response of the suspension. The motion of the free branches and non-percolating aggregates could be out of phase with the motion of the suspending liquid, so both viscous and elastic contributions are expected and the relative importance of each contribution will depend on the ratio of hydrodynamic to magnetic forces exerted on the aggregates. The behavior of the non-percolating aggregates and free branches is essentially similar in nature and described by the same equation of motion, so, we shall no more distinguish these two types of aggregates and call them pivoting aggregates, keeping in mind that the oscillations of the free branches could be restricted to lower amplitudes because of eventual collisions with percolating aggregates.

2. The percolating aggregates are supposed to be thin chains of fibers stacked together both by their extremities and their lateral surfaces. In such configuration, the fibers can easily slide on each other, therefore, the percolating aggregates are easily extensible under strain. The pivoting aggregates are supposed to be straight and rigid single chains of fibers. The structure observation [Fig. 1b] reveals that these chains may have different length but it is not obvious to determine the chain length distribution for the non-equilibrium thermodynamic state of the magnetic suspension in the absence of Brownian motion. At this stage, we consider that all the pivoting chains have the same length, $2 L$, equal to the half of the gap, $b$, between plates. Therefore, the chain length-to-diameter ratio - referred to as aspect ratio - is equal to: $p=b /(4 a)$, with $a$ being the fiber radius. This parameter is considered to be independent of the shear stress until a critical stress corresponding to the aggregate rupture by tensile hydrodynamic force. From the hydrodynamic point of view, the chains of fibers are assimilated to slender bodies and the Batchelor's slender body theory is used to describe their motion and generated stresses [37, 38].

3. To describe the quantities of particles and aggregates in the suspension, we introduce the volume fractions as follows: $\Phi$ is the volume fraction of particles in the suspension; $\phi$ is the relative volume fraction of the pivoting aggregates, i.e. the volume occupied by these aggregates divided by the total volume of all the aggregates; and $(1-\phi)$ is the relative volume fraction of percolating aggregates. $\phi$ is a free parameter of our model and could vary, in principle, between 0 and 1 . We assume that the relative volume fraction, $\phi$ of pivoting aggregates does not depend on the applied stress until some critical stress, $\sigma_{1}$, at which these aggregates become unstable and break (cf. Assumption \#4). At higher stresses, only pivoting aggregates exist and $\phi=1$. In other words, $\phi=\left\{\begin{array}{l}\text { adjustable parameter, } \sigma \leq \sigma_{1} \\ 1, \sigma>\sigma_{1}\end{array}\right.$ 
4. The suspension micro-structure is supposed to change with the increasing applied stress or strain, so, the five following sub-structures are expected. (I) At low-to-intermediate stresses, percolating clusters coexist with pivoting ones as explained above (Fig. 1c). (II) At some critical stress $\sigma_{1}$, the percolating aggregates become unstable, break in their middle point and are transformed into pivoting aggregates of the length corresponding approximately to the half of the gap between walls: $2 L \approx b / 2$ (Fig.1d). This instability has been discovered in our simulations and is reported in the next Section 3. Since the radius of thin percolating aggregates is of the order of the fiber radius, $a$, the secondary pivoting chains (formed due to the breakage of the percolating aggregates) will have the aspect ratio, approximately equal to: $p \approx b /(4 a)$, which is the same as the aspect ratio of the primary pivoting chains that existed before the breakage of the percolating chains. Thus, all the pivoting chains in this second aggregation regime are supposed to have approximately same size and aspect ratio, $p \approx b /(4 a)$. (III) Oscillating chains are subject to tensile hydrodynamic forces exerted along their axis. These forces tend to detach them from the walls. The particles situated on the chain extremity attached to the wall are supposed to be trapped in the wall rugosities. So, the chain detachment is expected to be accompanied by the rupture of the bonds between the trapped particles and the neighboring particles. Thus, the chains detach from the wall and become totally free when the hydrodynamic force becomes larger than the cohesive magnetic force between particles (Fig.1e). The onset of the regime of free chains occurs at the second critical stress $\sigma_{2}$ and is governed by the balance between these forces. (IV) Further increase of the stress, provokes an increase in hydrodynamic tensile forces, which destabilize the free chains and provoke their rupture in their middle part (Fig.1f). So, beyond the third critical stress, $\sigma_{3}$, the chain length is no more fixed but decreases progressively with increasing stress (or strain). We find that, at the same shear rate, the hydrodynamic force required to detach a chain from the wall is about four times the force required to destroy a free chain. Therefore, the $3^{\text {rd }}$ regime of unruptured free chains is expected to extend over a broad range of applied stresses, $\sigma_{2}<\sigma<\sigma_{3}$. (V) At high enough stresses, all the chains are completely destroyed and the suspension behaves as the one composed of isolated magnetic fibers (Fig.1g). The critical stress $\sigma_{4}$ corresponding to the onset of this regime is defined as the stress, at which the chain aspect ratio approaches to the one of a single fiber, namely, $p=7.6$.

5. In our model, we suppose that the percolating and pivoting aggregates do not slip on the walls. First, the magnetic field lines are concentrated in the rugosities of non-magnetic walls. This causes a strong adhesion of the aggregates to the walls, as pointed out by Laun et al. [39]. Second, the percolating aggregates are subject to tensile magnetic stresses, so the aggregates exert strong normal forces on rheometer walls [40]. Since the solid friction forces between the aggregate ends and the walls are directly proportional to the normal forces, we expect a strong wall friction, which could further enhance the adhesion between the aggregates and the walls.

6. Whatever the aggregation regime is, all the aggregates are supposed to oscillate in the shear xz-plane. The percolating aggregates in the first regime are always in phase with the strain, $\chi(t)$, and their orientation angle is defined as: $\tan \Theta=\gamma(t)$ [Fig.1c]. The pivoting and free 
chains are subject to the magnetic and hydrodynamic torques and make an angle $\theta(t)$ with the field direction, different from the angle $\Theta$ [Fig.1c].

7. The magnetic interactions between aggregates are taken into account by means of the mean field Maxwell-Garnett theory [41]. On the other hand, we neglect collisions and hydrodynamic interactions between aggregates in our fiber suspension of 5\% volume fraction for the reasons discussed in [12]. Nevertheless, the validity of this hypothesis will be revisited in the following section. Under the present assumptions, the balance of the hydrodynamic and magnetic torques acting on the non-percolated and free aggregates reads:

$$
\frac{8 \pi L^{3} f^{\perp}}{3 \ln (2 p)} \eta_{0}\left(\dot{\gamma} \cos ^{2} \theta-\dot{\theta}\right)=\frac{\chi_{f}{ }^{2}(1-\Phi)}{2+\chi_{f}(1-\Phi)} \mu_{0} H^{2} \cdot\left(2 \pi a^{2} L\right) \sin \theta \cos \theta
$$

The demonstration of the expression for the hydrodynamic torque (left-hand side of the Eq. (1)) together with the numerical factor $f^{\perp}$ is presented in Appendix, while the expression for the magnetic torque (right-hand side of the Eq. (1)) is given elsewhere [12]. The equation of motion of the non-percolated and free aggregates follows directly from Eq.(1):

$$
\dot{\theta}=\dot{\gamma} \cos ^{2} \theta-\frac{1}{\tau(\theta)} \sin \theta \cos \theta
$$

and can be rewritten in the alternative form, as follows:

$$
\frac{\mathrm{d} \tan \theta}{\mathrm{d} t}+\frac{\tan \theta}{\tau(\theta)}=\dot{\gamma}
$$

where $\tau(\theta)$ is the relaxation time, corresponding to a characteristic time required for the reorientation of the aggregate along the field direction in a quiescent fluid, and defined by the following equation:

$$
\tau(\theta)=\frac{2+\chi_{f}(1-\Phi)}{\chi_{f}{ }^{2}(1-\Phi)} \cdot \frac{4 p^{2} f^{\perp}}{3 \ln (2 p)} \cdot \frac{\eta_{0} \mu^{2}(\theta)}{\mu_{0} H_{0}^{2}}
$$

In these equations, $\eta_{0}$ is the dynamic viscosity of the suspending liquid, $\mu_{0}=4 \pi \cdot 10^{-7} \mathrm{H} / \mathrm{m}$ is the magnetic permeability of vacuum, $\chi_{f}$ is the magnetic susceptibility of fibers, $H$ is the intensity of the magnetic field inside the fiber suspension sandwiched between two plates. Because of the demagnetizing effect, this field is smaller by the factor $\mu$ than the external magnetic field, $H_{0}$, namely, $H=H_{0} / \mu$, where $\mu$ is the relative magnetic permeability of the fiber suspension. The latter is the function of the aggregates' orientations and is defined using the MaxwellGarnet theory. In the general case, covering all aggregation regimes, the expression for the magnetic permeability $\mu(\theta)$ reads:

$$
\mu(\theta)=\phi\left[\mu_{\|} \cos ^{2} \theta+\mu_{\perp} \sin ^{2} \theta\right]+(1-\phi)\left[\mu_{\|} \frac{1}{\left(1+\gamma^{2}\right)^{2}}+\mu_{\perp} \frac{\gamma^{2}}{\left(1+\gamma^{2}\right)^{2}}\right],
$$


where $\mu_{\|}=1+\Phi \chi_{f}$ and $\mu_{\perp}=\left[2+\chi_{f}(1+\Phi)\right] /\left[2+\chi_{f}(1-\Phi)\right]$ are the components of the suspension magnetic permeability along the major and minor axes of aggregates, the factors $1 /\left(1+\gamma^{2}\right)^{2}$ and $\gamma^{2} /\left(1+\gamma^{2}\right)^{2}$ correspond to $\cos ^{2} \Theta$ and $\sin ^{2} \Theta$, respectively, with $\Theta$ - the orientation angle of percolating aggregates (Fig. 1c). The first term of this equation stands for the contribution of the pivoting and free chains to the magnetic permeability and the second term - to the contribution of the percolating aggregates. In the four last aggregation regimes, percolating aggregates no more exist, so, the second term of Eq. (5) disappears and $\phi$ is taken to be 1 in the first term. Note that, since the magnetic permeability depends on the chain orientation angle $\theta$, the relaxation time $\tau$ also depends on it, and, so, does not remain constant over the oscillation period. We can consider $\tau$ to be constant in the case of small amplitude oscillations.

The hydrodynamic torque acting on pivoting chains appears to be roughly four times the torque acting on free chains of the same size and at the same shear rate. Therefore the relaxation time is about four times larger for pivoting chains as compared to free chains. The time $\tau$ is defined by the same Eq. (4) for both chains but the numerical factor $f^{\perp}$ is different, as pointed out in Appendix.

In three first aggregation regimes (Figs.1c-e), the aspect ratio of the pivoting and free unruptured chains is fixed and equal to: $p=b /(4 a)$. In the regime of the free ruptured chains (Fig.1d), their aspect ratio is governed by the balance of hydrodynamic tensile and magnetic forces, the expressions for these forces read:

$$
\begin{aligned}
& F_{h}=\frac{\pi L^{2} f_{2}^{\|}}{\ln (2 p)} \eta_{0} \dot{\gamma} \sin \theta \cos \theta, \\
& F_{m}=2 \pi \mu_{0} M_{S} H a^{2} \cos ^{2} \theta
\end{aligned}
$$

where $M_{S}$ is the saturation magnetization of magnetic fibers, $f_{2}^{\|}=(1+0.5 \varepsilon) /(1-1.5 \varepsilon)$. The magnetic force [Eq.(7)], consolidating the chains is the attractive force acting between two contacting fibers. This force was calculated using the Ginder's approach considering saturation effects in touching magnetic particles [42], as specified in details in [12]. In our model we consider that over a given oscillation period the free chain keeps the same length. Both the magnetic and hydrodynamic forces vary during the period of the chain oscillation. The chains are considered to be stable when the hydrodynamic tensile force is smaller than the magnetic force. But if, at a certain orientation angle, $\theta$, the hydrodynamic force becomes larger than the magnetic one, the chains are supposed to break into two parts. We suppose that the rupture occurs when the maximum ratio of both forces, over the period, is equal to unity, namely: $\max \left(F_{h} / F_{m}\right)=1$. Thus, by means of the Eqs. (6), (7), the expression for the aspect ratio of the ruptured free chains reads: 


$$
\left[\frac{p^{2} f_{2}^{\|}}{\ln (2 p)}\right]_{\text {ruptured }}=\frac{2 \mu_{0} M_{S} H_{0}}{\eta_{0} \cdot \max [\mu \dot{\gamma} \tan \theta]}
$$

Here we have taken into account that the internal magnetic field is $H=H_{0} / \mu$.

Note that the same force balance [Eqs. (6)-(8)] holds for determining the critical stress $\sigma_{2}$ corresponding to transition between the regimes of pivoting and free chains (the stress, at which the pivoting chains are ruptured from the walls). In this case, we have only to replace in Eqs. (6), (8) the numerical factor $f_{2}^{\|}$by appropriate value $f_{2}^{\|}=4(1+0.307 \varepsilon) /(1-0.5 \varepsilon)$ and take the aspect ratio fixed and equal to $p=b /(4 a)$. The critical stress $\sigma_{2}$ intervenes implicitly into the Eq. (8) through the product, $\mu \dot{\gamma} \tan \theta$, which is found by solving simultaneously Eqs. (3), (9).

In the most general case, the shear stress developed in our fiber suspension has a contribution from the suspending liquid, $\sigma_{s}$, a contribution from percolating aggregates, $\sigma_{p}$, and a contribution from pivoting or free ones, $\sigma_{f-p}$, the two last weighed by the appropriate volume fraction, $(1-\phi)$ and $\phi$, respectively, such that the total stress is

$$
\begin{aligned}
& \sigma=\sigma_{s}+\sigma_{f-p}+\sigma_{p}=\eta_{0} \dot{\gamma}+\phi\left[\Phi \eta_{0} \dot{\gamma}\left(2+\frac{2 p^{2} f_{1}^{\|}}{3 \ln (2 p)} \sin ^{2} \theta \cos ^{2} \theta\right)+\right. \\
& \left.+\Phi \mu_{0} H^{2} \frac{\chi_{f}^{2}(1-\Phi)}{2+\chi_{f}(1-\Phi)} \sin \theta \cos ^{3} \theta\right]+(1-\phi) \cdot \Phi \mu_{0} H^{2} \frac{\chi_{f}^{2}(1-\Phi)}{2+\chi_{f}(1-\Phi)} \cdot \frac{\gamma}{\left(1+\gamma^{2}\right)^{2}}
\end{aligned}
$$

where the factor $\gamma /\left(1+\gamma^{2}\right)^{2}$ corresponds to $\sin \Theta \cos ^{3} \Theta$ for percolating aggregates and $f_{1}^{\|}=(1+0.64 \varepsilon) /(1-1.5 \varepsilon), \varepsilon=1 / \ln (2 p)$. In the Eq. (9), the contribution from pivoting or free chains, $\sigma_{f-p}$, has both hydrodynamic and magnetic terms, while the contribution from the percolating aggregates, $\sigma_{p}$, has only magnetic term arisen from the restoring magnetic torque. The hydrodynamic term is absent in $\sigma_{p}$ because the percolating aggregates move affinelly with the suspending liquid. In particular, these aggregates are supposed to be very thin (aspect ratio about 40), so at any point on the aggregate surface, there is no velocity difference between the solid and liquid phases. McLeish et al. [33] have also neglected the viscous dissipation coming from percolating chains and obtained a reasonable correspondence with experiments for the frequency dependence of the storage modulus. The last term of the Eq. (9) appears only in the first aggregation regime. In the last four regimes, the percolating aggregates are absent, and $\phi=1$.

The Eqs. (3)-(5), (8), (9) for the regime of free ruptured chains or (3)-(5), (9) for other four regimes form a closed system, which is solved under the initial conditions, as follows: $\theta(0)=0$ and $\chi(0)=0$. Both the strain, $\chi(t)$ and the angle $\theta(t)$ are unknown functions, so, by change of variables, $\Psi(t)=\int_{0}^{t}(\tan \theta / \tau) \mathrm{d} t$, we reduce a pair of differential equations (3), (9) into a single 
second order differential equation with respect to the function $\Psi(t)$. Once this function is determined, the unknowns, $\theta(t)$ and $\chi(t)$ are found as: $\tan \theta=\tau \dot{\Psi}$ and $\gamma=\tau \dot{\Psi}+\Psi$. This trick is, however, used only for the first regime of coexisting percolating and pivoting aggregates. In the other four regimes, we solve the Eq.(9) directly for $\theta(t)$, after having replaced $\dot{\gamma}$ by the left-hand side of the Eq.(3).

At the next step, the output signal, $\not(t)$, is expanded into Fourier series as follows [43]:

$$
\gamma(t)=\sigma_{0} \sum_{n}\left\{J_{n}{ }^{\prime}\left(\sigma_{0}, \omega\right) \cos (n \omega t)+J_{n}{ }^{\prime}\left(\sigma_{0}, \omega\right) \sin (n \omega t)\right\}
$$

where $J_{n}, J_{n}$ " are the $n$-th harmonic real and imaginary part of the compliance, respectively. In our study, we use the fundamental shear moduli, $G_{1}$ ' and $G_{1}$ " as the principle characteristic of the fiber suspension viscoelasticity. These quantities are related to the compliance through the following expressions:

$$
G_{1}{ }^{\prime}=\frac{J_{1}{ }^{\prime}}{J_{1}{ }^{\prime 2}+J_{1}{ }^{\prime 2}}, \quad G_{1}{ }^{\prime \prime}=\frac{J_{1}{ }^{\prime}}{J_{1}{ }^{2}+J_{1}{ }^{12}}
$$

In the plate-plate geometry used in our experiments, the shear strain depends on the radial coordinate of the geometry, therefore, we must perform the Mooney correction for the stress amplitude, $\sigma_{0}$, and for the shear moduli. The relation between the amplitude of the apparent stress, $\sigma_{a}$, measured by a rheometer, and the amplitude of the strain, $\gamma_{R}$, measured at the border of the rotating plate, takes the form [44]:

$$
\sigma_{a}=\frac{4}{\gamma_{R}^{3}} \int_{0}^{\gamma_{R}} \sigma_{0} \gamma_{0}^{2} \mathrm{~d} \gamma_{0}
$$

In the present paper, we consider the stress controlled mode of the oscillatory shear. So, in order to perform the Mooney correction, first, we calculate the strain amplitude $\gamma_{0}$ as function of the non-corrected stress $\sigma_{0}$ with the help of the Eq. (10), then, we make a polynomial fit to $\sigma_{0}$ versus $\gamma_{0}$ dependence and finally we perform integration [Eq. (12)]. The shear moduli are calculated using the Eq. (11) and subsequently corrected by a multiplier $\sigma_{a} / \sigma_{0}$. All results presented along this paper refer to the corrected quantities.

We shall now analyze our theoretical and experimental results on the shear moduli of the suspension as well as on the output signal waveforms.

\section{Results and discussion}

\subsection{Shear moduli}

Experimental dependencies of the shear moduli, $G_{1}$ ' and $G_{1}$ " on the stress amplitude, $\sigma_{0}$, are shown in Figs.2a,b for the excitation frequency, $f=1 \mathrm{~Hz}$ and for six values of the external magnetic field, $H_{0}$. In all cases, both moduli increase with the growth in the magnetic field 
intensity and decrease with the stress amplitude. In particular, a short linear viscoelastic plateau at $\sigma_{0} \lesssim 1 \mathrm{~Pa}$ is followed by a rapid decrease of the moduli until a second quasi-plateau, which is better distinguished for the loss modulus curves. After this second quasi-plateau, there is a second abrupt decrease of the moduli, at the end of which the storage modulus shows the third final plateau after some local minimum.
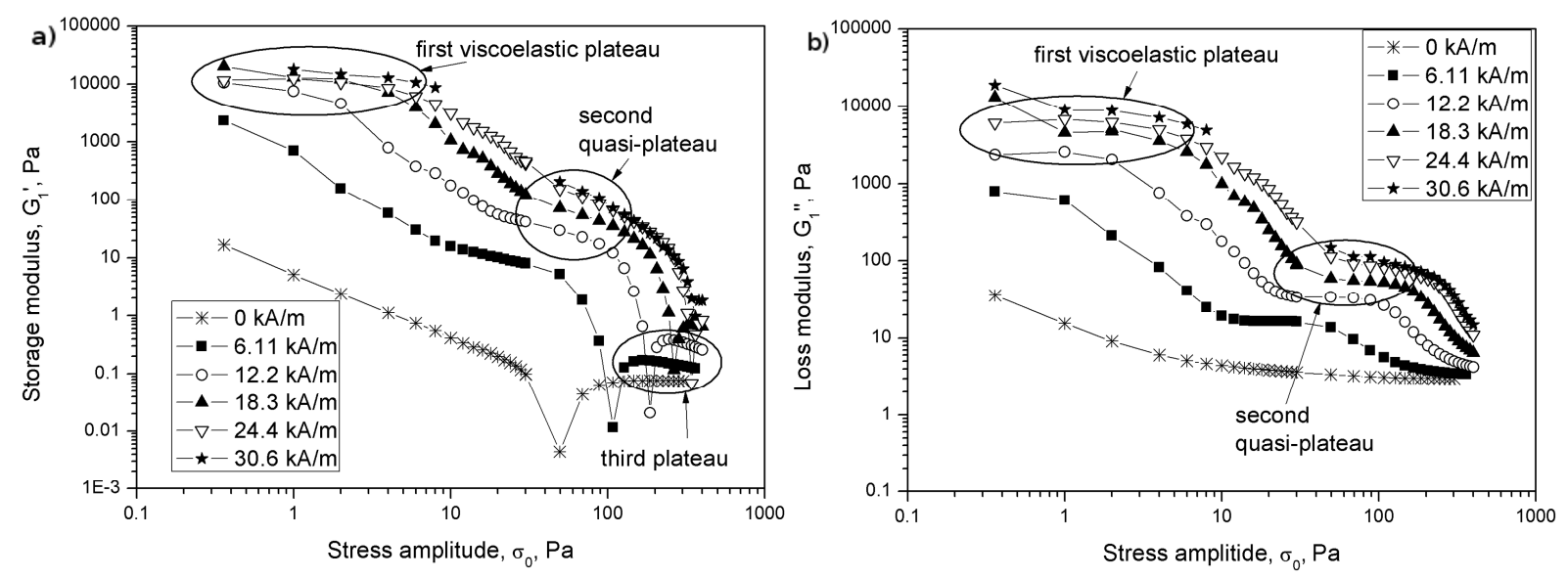

Fig.2. Experimental stress dependencies of the storage (a) and loss (b) moduli at the excitation frequency of $1 \mathrm{~Hz}$

The first viscoelastic plateau, which appears only for the magnetic fields $H_{0} \geq 12.2 \mathrm{kA} / \mathrm{m}$, corresponds to the strain amplitudes, $\gamma_{0}$, as low as $10^{-4}-10^{-3}$. At such strains, the upper plate displacement during an oscillation cycle is as small as 20-200 nm, i.e. much smaller than the fiber's minor dimension - diameter $2 a=4.9 \mu \mathrm{m}$. Thus, we cannot expect a homogenous deformation of the aggregates, but rather a rearrangement of fibers inside the aggregates accompanied by their microscopic displacement and/or by their elastic bending. The latter could explain high values of the storage modulus at small amplitudes (more than $10 \mathrm{kPa}$ at the particle volume fraction of 5\%). The large values of the loss modulus could come from the non-affinity of the fiber displacement on microscopic scale, as pointed out by Klingenberg [34]. The first decrease of the storage moduli followed by a second quasi-plateau probably corresponds to a gradual transition from microscopic-to-macroscopic scale deformation of the suspension structure. At the end of this transition, the percolating aggregates are expected to be strained uniformly at small but measurable angles. Actually, at the magnetic field intensity, $H_{0}=30.6 \mathrm{kA} / \mathrm{m}$, the second quasi-plateau starts at $\gamma_{0} \approx 0.1$ corresponding to the upper plate displacement of $20 \mu \mathrm{m}$, which is at least, five times the fiber diameter. This second quasiplateau is attributed to the second quasi-linear viscoelastic regime governed only by macroscopic deformations of the structure. Starting from this quasi-plateau, we can safely apply our theory. For the better comparison with experiments, we plot both experimental and theoretical dependencies $G_{1}{ }^{\prime}\left(\sigma_{0}\right), G_{1}{ }^{\prime}\left(\sigma_{0}\right)$ in Fig.3 for the magnetic field intensity $H_{0}=30.6 \mathrm{kA} / \mathrm{m}$ and consider the stress range $\sigma_{0}>30 \mathrm{~Pa}$ corresponding to the beginning of the second quasi-plateau. 


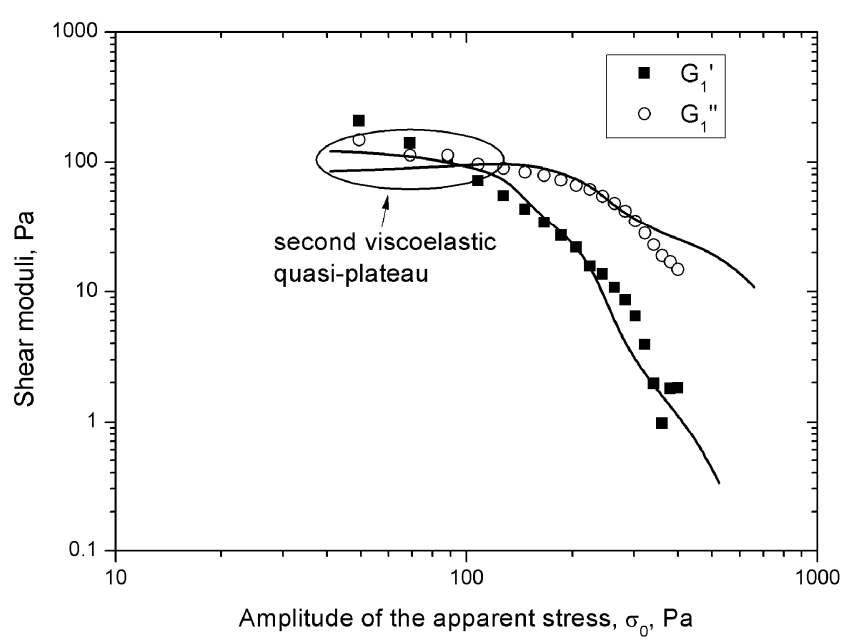

Fig.3. Comparison theory-experiments for the stress-dependence of the shear moduli at the magnetic field intensity, $H_{0}=30.6 \mathrm{kA} / \mathrm{m}$ and frequency $f=1 \mathrm{~Hz}$. The fit parameter of the model is chosen to be $\phi=0.7$. Solid lines correspond to calculations and points - to experimental results.

The best correspondence between theory and experiments is achieved for the values of the fitting parameter equal to $\phi=0.7$. This parameter is kept the same throughout all our simulations. Nevertheless, in the broad range of $\phi(0.5<\phi<1)$, the calculated shear moduli differed not more than two times from the values reported in Fig.3.

After a second viscoelastic quasi-plateau (which extends from $\sigma_{0} \approx 40 \mathrm{~Pa}$ to $\sigma_{0} \approx 100 \mathrm{~Pa}$ ), a more gradual decrease in shear moduli is caused first by an abrupt increase in oscillation amplitude of the aggregates and, second, by their rupture starting from the critical stress equal to $430 \mathrm{~Pa}$ in our theory. The storage modulus is subject to a more drastic decrease than the loss modulus. The crossover of both moduli occurs at $\sigma_{0} \approx 100 \mathrm{~Pa}$ and is well captured by our model. In our calculations, we did not reproduce the small local minimum of the storage modulus at $\sigma_{0} \approx 350 \mathrm{~Pa}$ [Fig.3]. A small increase of the storage modulus after this local minimum could occur because of the short-range hydrodynamic interactions and collisions between aggregates, which would restrict the aggregate motion to smaller amplitudes. Note that, apart from this local minimum, both experimental and theoretical curves $G_{1}{ }^{\prime}\left(\sigma_{0}\right)$, $G_{1}$ " $\left(\sigma_{0}\right)$ are relatively smooth in the whole range of the applied stresses, thus, the transitions between the five aggregation regimes are not clearly distinguishable in these curves.

However, the transition between the regime of coexisting percolating and pivoting aggregates [Fig.1c] to the regime of purely pivoting aggregates [Fig.1d] requires a special attention. At $\sigma_{0}>140 \mathrm{~Pa}$, the solution for the strain $\chi(t)$ becomes strongly asymmetric relative to the equilibrium position, $\gamma=0$, which does not have any physical sense. The percolating clusters are considered to be unstable and they are supposed to break in the middle, remaining attached to one of the walls. So, they transform into pivoting aggregates, which, according to our calculations, are stable in the broad range of applied stress. Note that this instability is the one, which defines the static yield stress through its maximum versus applied strain [45]. 
It is worth mentioning that, in the limit of small deformations, $\gamma \ll 1, \theta \ll 1$, our model admits the following analytical solution for the shear moduli at the second viscoelastic plateau:

$$
\begin{aligned}
& G_{1}{ }^{\prime}=\left(G_{1}{ }^{\prime}\right)_{f-p}+\left(G_{1}{ }^{\prime}\right)_{p}=\phi \cdot \Phi \mu_{0} H^{2} \frac{\chi_{f}^{2}(1-\Phi)}{2+\chi_{f}(1-\Phi)} \cdot \frac{(\omega \tau)^{2}}{1+(\omega \tau)^{2}}+(1-\phi) \cdot \Phi \mu_{0} H^{2} \frac{\chi_{f}^{2}(1-\Phi)}{2+\chi_{f}(1-\Phi)}(13) \\
& G_{1}{ }^{\prime \prime}=\left(G_{1}{ }^{\prime \prime}\right)_{f-p}+\left(G_{1}{ }^{\prime \prime}\right)_{s}=\phi \cdot \Phi \mu_{0} H^{2} \frac{\chi_{f}^{2}(1-\Phi)}{2+\chi_{f}(1-\Phi)} \cdot \frac{\omega \tau}{1+(\omega \tau)^{2}}+2 \phi \frac{\Phi}{\Phi_{a}} \eta_{0} \omega+\eta_{0} \omega
\end{aligned}
$$

Here, the $\left(G_{1}{ }^{\prime}\right)_{f-p}$ and $\left(G_{1}{ }^{\prime}\right)_{p}$ terms in the expression for $G_{1}$ 'stand for the elastic contributions of the pivoting/free and percolating aggregates, respectively. The loss modulus $G_{1}$ " has only a viscous contribution $\left(G_{1} \text { " }\right)_{f-p}$ from pivoting/free chains (first two terms) and a solvent contribution, $\left(G_{1}{ }^{\prime}\right)_{s}=\eta_{0} \omega$. The term $2 \phi\left(\Phi / \Phi_{a}\right) \eta_{0} \omega$ arises from the general expression for the viscous stress tensor of anisotropic particle suspensions [46, 47] and corresponds to the viscous dissipation due to eventual rotations of the chains around their major axis when they are slightly shifted from the shear plane. According to Eqs. (13), (14), the resultant response of the system of percolating and pivoting aggregates has features of the Maxwell-like and Kelvin-Voight behaviors. The former is provided by pivoting (or free) chains with the relaxation time $\tau$, and the latter comes from percolating aggregates, which contribution to the storage modulus is frequency-independent. The resultant frequency behavior, predicted by Eqs. (13), (14) for the linear viscoelastic regime, is illustrated in Fig.4a. The low-frequency plateau of the storage modulus (solid curve) is due to the elastic response of the percolating aggregates. At growing frequencies, pivoting chains give a supplementary contribution to the storage modulus, which becomes frequency independent in the limit $(\omega \tau) \gg 1$ and provides the high-frequency plateau of $G_{1}$ '. The loss modulus increases linearly with $\omega$ in the lowfrequency limit. At high frequencies, pivoting chains contribute to a decrease of the loss modulus $\left(\left(G_{1} "\right)_{f-p} \sim(\omega \tau)^{-1}\right)$, but the solvent contribution remains always increasing. So, the theoretical $\mathrm{N}$-shape of the frequency dependence of the loss modulus is explained by a competition between both contributions, such that, in the limit $(\omega \tau) \gg 1$, the aggregate contribution is shadowed by the solvent one and loss modulus again increases linearly with the frequency. The experimental data, obtained for a relatively low stress, $\sigma_{0}=30 \mathrm{~Pa}$, are not in a good correspondence with our model. A possible reason for such discrepancy is that, at this stress, a microscopic-scale rearrangement of fibers inside the aggregates is still important. However, both in experiments and in theory, we find a high-frequency plateau of the storage modulus and do not observe an important decrease of the loss modulus, as reported by McLeish et al. [33]. Perhaps, this is because in their study, the authors did not achieve the frequencies high enough to produce significant viscous response of the solvent. Since our theory is more relevant for large oscillation amplitudes, let us inspect now the frequency dependence of the shear moduli at large stress, $\sigma_{0}=244 \mathrm{~Pa}$ [Fig.4b]. 

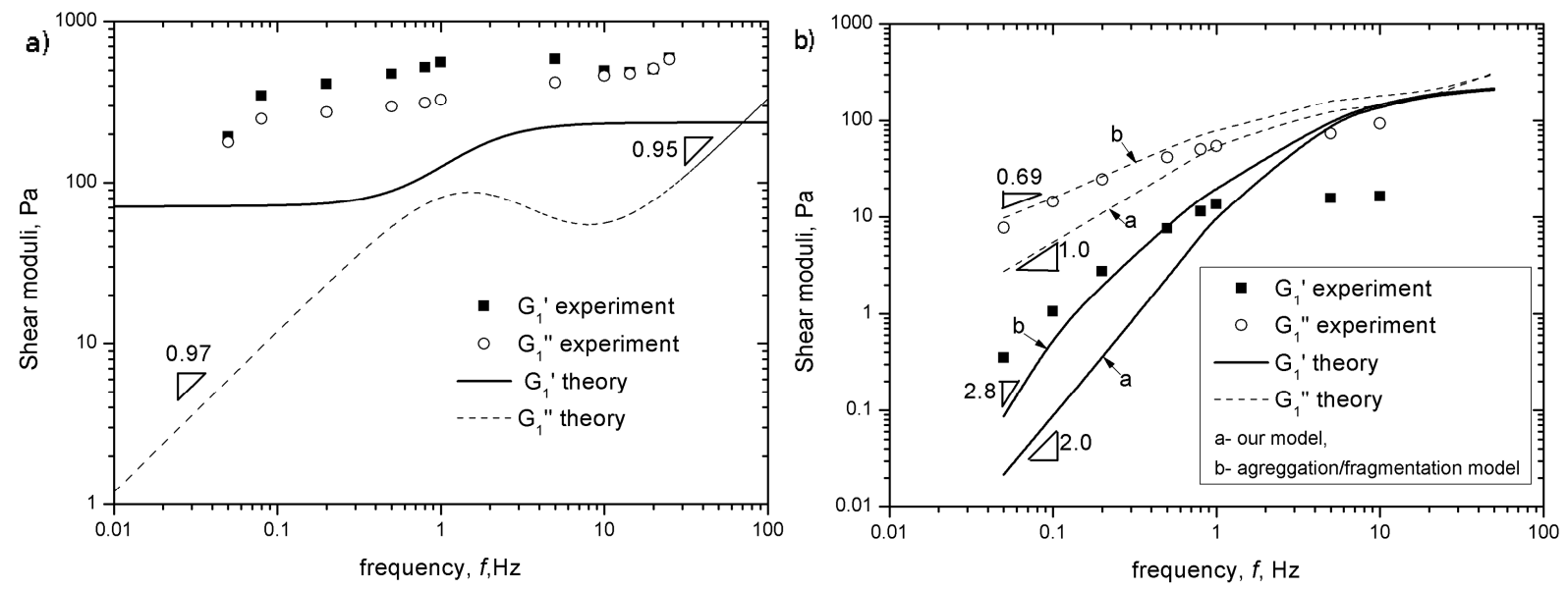

Fig.4. Frequency dependence of the shear moduli in the linear (a) and nonlinear (b) viscoelastic regimes. The stress amplitude is $\sigma_{0}=30 \mathrm{~Pa}$ in the first case (a) and $244 \mathrm{~Pa}$ in the second case (b). In both cases, the magnetic field intensity is $H_{0}=30.6 \mathrm{kA} / \mathrm{m}$.

We see that our theory (curves denoted by "a" in Fig. 4b) predicts a similar behavior as the one observed in experiments, however, the quantitative agreement is worse than for the stress dependence of the shear moduli. According to calculations, at the given stress, $\sigma_{0}=244 \mathrm{~Pa}$, the suspension develops the second aggregation regime - regime of pivoting chains, within the whole frequency range considered $(0.05 \mathrm{~Hz}<f<50 \mathrm{~Hz})$. Since the percolating aggregates are absent, we do not observe a low-frequency plateau of the storage modulus [Fig.4b]. In theory, the latter increases as $\omega^{2}$ at low frequencies and approaches a plateau at $f>10 \mathrm{~Hz}$. Such behavior of the storage modulus is relevant for the Maxwell viscoelastic response, as discussed above. In experiments, $G_{1}$ ' shows a weaker dependence at low frequencies $\left(G_{1}{ }^{\prime} \propto \omega^{1.34}\right)$ and the final plateau is shifted to lower frequency. The loss modulus also grows monotonically with the frequency (as $\omega^{1}$ in theory and $\omega^{0.73}$ in experiments) and does not show any local minimum as the one predicted for linear viscoelastic regime. Apart from a solvent contribution, a supplementary hydrodynamic contribution from aggregates appears at high stresses. Thus, the maximum of the $G_{1}$ ' $(f)$ curve might be suppressed by these hydrodynamic terms, both increasing with the frequency. Note finally that the loss modulus appears to be larger than the storage modulus within the whole frequency domain, that stands for the liquid-like behavior of the fiber suspension at the given stress amplitude and the given magnetic field.

\subsection{Supplementary characteristics of non-linear viscoelasticity}

Another important characteristic of the LAOS response of the fiber suspension is the dependency of the stress amplitude on the strain amplitude, shown in Fig.5 for the magnetic field intensity $H_{0}=30.6 \mathrm{kA} / \mathrm{m}$ and the frequency $f=1 \mathrm{~Hz}$. This dependency shows clearly a strain softening behavior of the fiber suspension with the progressive decrease in the slope. At increasing stress, the oscillation amplitude increases, and the suspension structure becomes less rigid. First, the aggregates detach from the wall, then they are ruptured by the tensile force exerted by the ambient liquid. This should explain the strain softening observed. In the 
present case of the controlled stress (solid line and points in Fig.5), the $\sigma_{0}\left(\gamma_{0}\right)$-curve shows a power-law dependence at the strain amplitudes $\gamma_{0}<3$ and becomes quasi-linear at $\gamma_{0}>5$. We observe a fairly good correspondence between theory and experiments at the applied stress up to $300 \mathrm{~Pa}$. Above this value, our theory (solid curve) underestimates the strain amplitude and predicts a stronger elastic response. Such discrepancy could come from the overestimation of the length of chains subject to tensile hydrodynamic force.

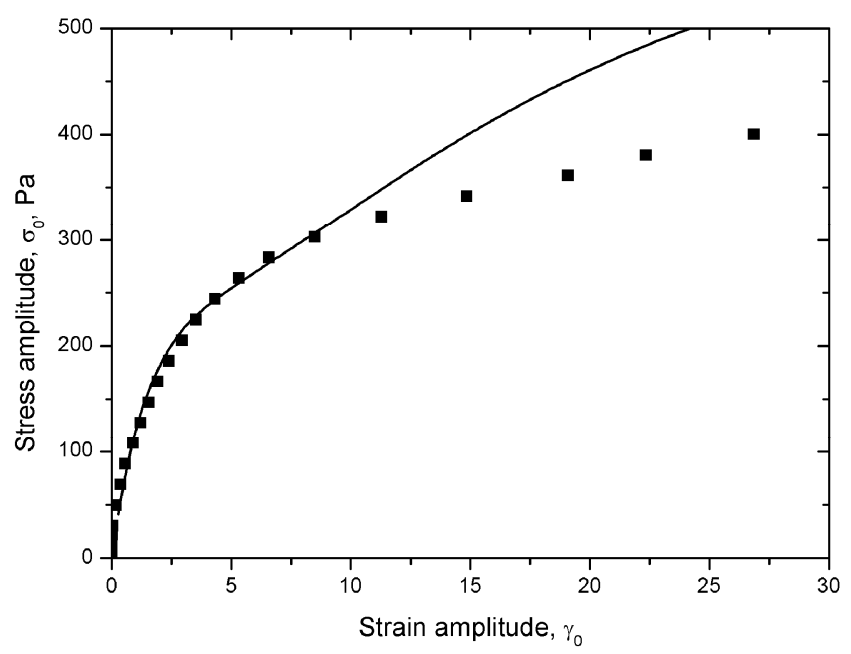

Fig.5. Stress amplitude, $\sigma_{0}$, versus strain amplitude, $\gamma_{0}$. Line stands for the theory, points - for experiments. The magnetic field intensity is $H_{0}=30.6 \mathrm{KA} / \mathrm{m}$ and the excitation frequency is $f=1 \mathrm{~Hz}$.

Useful information on the non-linear viscoelastic response can be provided by the waveforms and Fourier analysis of the output strain. In Fig.6, we present a set of the plots, including strain waveforms, Lissajous plots and harmonic analysis of the strain waveforms, for the frequency $f=1 \mathrm{~Hz}$, magnetic field intensity $H_{0}=30.6 \mathrm{kA} / \mathrm{m}$ and for the three values of the applied stress amplitude, $\sigma_{0}=127,244$ and $342 \mathrm{~Pa}$. These values correspond to three first aggregation regimes: regime of co-existing percolating and pivoting aggregates (Fig.1c), regime of pivoting chains (Fig.1d) and regime of free non-ruptured chains (Fig.1e). 

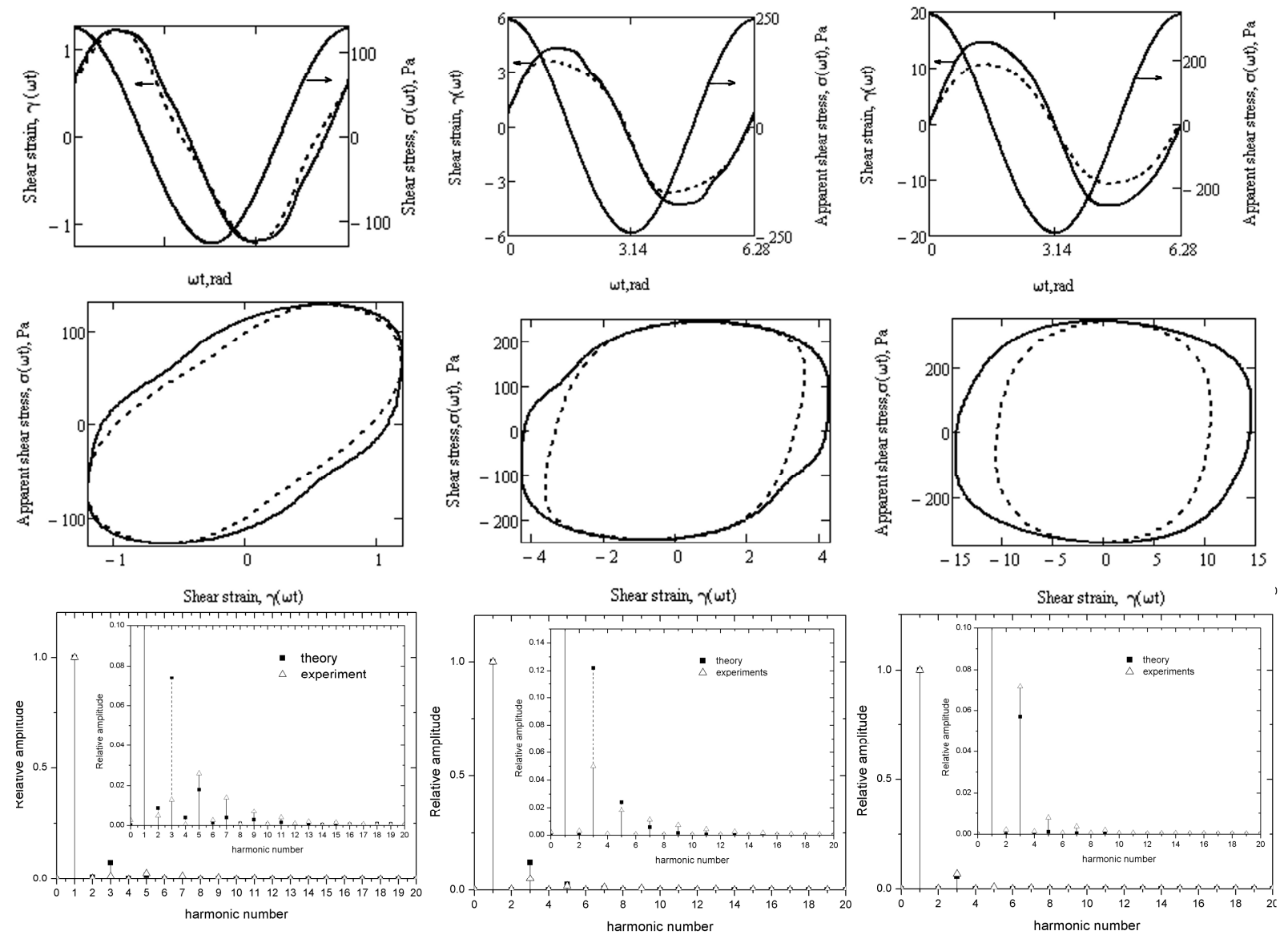

Fig.6. From top-to-bottom: strain waveforms (solid line - experiments, dash line - theory); Lissajous plots (solid line - experiments, dash line - theory); Fourier analysis of the strain waveforms. All the data are collected for the excitation frequency $f=1 \mathrm{~Hz}$, the magnetic field intensity $H_{0}=30.6 \mathrm{kA} / \mathrm{m}$ and for the three values of the amplitude $\sigma_{0}$ of the applied stress: $127 \mathrm{~Pa}$ (left column), $244 \mathrm{~Pa}$ (middle column) and $342 \mathrm{~Pa}$ (right column).

The strain waveforms show an important phase-shift with respect to the applied stress [first row in Fig. 6]. This phase-shift increases with increasing stress, signifying a progressive transition from solid-to-liquid-like behavior, which is consistent with the stress dependence of the shear moduli [Figs.2, 3]. The measured waveforms (solid lines) are non-sinusoidal that correspond to the nonlinear viscoelastic response of our suspension, and our theory (dashed lines) reproduces these waveforms reasonably well. Note that analysis of the output waveforms can only be reliable at a low noise level or at a high signal-to-noise ratio. For the three values of the applied stress shown in Fig.6, the signal-to-noise ratio is estimated as the square of the signal amplitude -to noise amplitude ratio and is found to be about $10^{4}$, or $40 \mathrm{~dB}$ in decibel units.

The viscoelastic non-linearity can also be visualized in Lissajous plots, $\sigma(t)$ versus $\chi(t)$ [second row of the Fig.6]. We should keep in mind that this analysis can only be efficient at moderate-to-high levels of non-linearity. At the stress amplitude, $\sigma_{0}=127 \mathrm{~Pa}$, a near-oval shape of the Lissajous plot is observed both in experiments (solid curve) and in theory (dashed curve). This shape indicates that, at the considered stress, we are still not far from the linear viscoelastic regime. The viscoelastic state at $\sigma_{0}=127 \mathrm{~Pa}$ belongs to the end part of the 
viscoelastic quasi-plateau [Fig.3]. At higher stress, the Lissajous plots become more compressed, their shape tends to a rounded-end rectangular one at $\sigma_{0}=342 \mathrm{~Pa}$. Such a shape could suggest a viscoplastic behavior of the suspension: at growing strains, the stress remains nearly constant until the structure yields and the stress is rapidly released when the strain direction is reversed. A similar explanation has been given by Parthasarathy and Klingenberg [27] for the LAOS response of ER fluids, however, the shape of their Lissajous plots were much noisier than the one reported in Fig.6. Note finally, that we obtain a reasonable correspondence between measured (solid lines) and calculated (dashed lines) Lissajous plots, even though our theory underestimates the strain amplitude at high stresses (244 and 342Pa).

The non-linearity of the viscoelastic response can be better quantified by the amplitude of the higher harmonics of the strain signal. We have applied Fourier transform to both theoretical and measured $\chi(t)$-signal following the Eq. (10) and reported the amplitude of each harmonic normalized by the first harmonic amplitude in Fig.6 (bottom row of figures). The normalized amplitudes of the higher harmonics were calculated using the following formula: $A_{n}=\left[\left(J_{n}^{\prime}\right)^{2}+\left(J_{n}^{\prime \prime}\right)^{2}\right]^{1 / 2} /\left[\left(J_{1}^{\prime}\right)^{2}+\left(J_{1}^{\prime \prime}\right)^{2}\right]^{1 / 2}$. First, we see that the even harmonics are generally much less important than the odd harmonics and they are attributed to a noise in the processed signals. The smallness of even harmonics supports the fact that the viscoelastic response of the suspension must be symmetric with respect to shear strain and shear rate directions [48].This can also suggest the absence of the wall slip in the system, which confirms the assumption \#5 of the Section 3. Second, the relative amplitude of the harmonics is generally a decreasing function of the harmonic number such that the $9^{\text {th }}$ and higher harmonics become indiscernible from noise.

A more detailed analysis of the viscoelastic non-linearity can be obtained by plotting the stress-dependence of amplitudes of the second and the third harmonics of the output strain (Fig.7). We plotted this graph for the stress amplitudes $\sigma_{0}>50 \mathrm{~Pa}$, at which the ratio of the $3^{\text {rd }}$ harmonic amplitude to noise amplitude is higher than 10. For the given stress range, the experimental amplitude $A_{3}$ of the $3^{\text {rd }}$ harmonic increases monotonically with the stress. This suggests an increase of the viscoelastic non-linearity with the growth in the excitation amplitude. In theory, the relative amplitude of the $3^{\text {rd }}$ harmonic is non-monotonic function of the stress amplitude. However, within each aggregation regime, the theoretical value of $A_{3}$ remains a growing function of the applied stress but the transition to a next aggregation regime is accompanied with a jumped decrease in $A_{3}$. Note that both theoretical and experimental values of the relative amplitude of the $3^{\text {rd }}$ harmonic does not exceed $12 \%$, so the eventual jumps in $A_{3}$ do not lead to visible jumps of the stress-strain dependence (Fig. 5). The theoretical results on $A_{3}$ agree with experimental ones only for the aggregation regimes of free non-ruptured and free ruptured chains $(\sigma>230 \mathrm{~Pa})$. At lower stresses the theory fails to reproduce correctly the values of $A_{3}$. Such discrepancy at low to moderate stress does not contradict to a good agreement between theoretical and experimental waveforms. The later agreement comes from the fact that the theory predicts the fundamental harmonic reasonably well. So, the discrepancy in higher harmonics (which are less important) does not influence a lot a good correspondence between the output waveforms. Finally, we see in Fig. 7 that the 
relative amplitude of the experimental $2^{\text {nd }}$ harmonic $A_{2}$ does not exceed the noise level in the whole range of the stresses, which is in agreement with our theory.

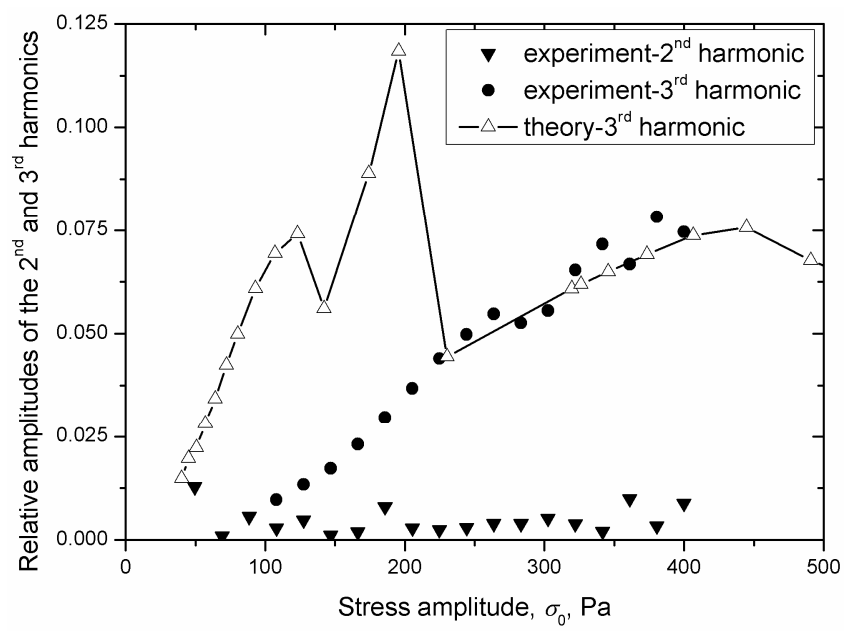

Fig.7. Stress-dependence of the relative amplitudes of the $2^{\text {nd }}$ and the $3^{\text {rd }}$ harmonics of the output strain signal

\subsection{Discussion in view of existing models}

As already mentioned, our theory bears some features of McLeish's string model of ER fluids [33]. Recall that in their model, the coexistence of percolated single chains with nonpercolated flexible chains has been supposed. First, the percolating chains were deformed affinelly and extended by the shear along their axis, such that the particles lost mechanical contacts between them and the spacing between particles increased with increasing strain. We consider this assumption to be unrealistic because, once stacked together, the particles will not separate until the chain is broken by an external (hydrodynamic) force. It is more likely that the particles form column structures that can be easily extended without loss of interparticle contacts. Second, in McLeish's model, pivoting chains were supposed to be flexible and develop an infinite series of bending modes, each associated with appropriate relaxation time. In fiber suspension, the fibers composing the pivoting and free chains are likely stacked together by their lateral surfaces. So, these chains are supposed to be stiffer and less compliant to the bending compared to single chains of spherical particles. Therefore, we assumed our aggregates to be rigid, such that the suspension response was described by a single relaxation time. Surprisingly, at large amplitudes, our simple model gave a reasonable correspondence with experiments. At last, McLeish et al. [33] supposed a minor fraction, $\phi$, of the pivoting chains, of the order of a few percent relative to the total quantity of the solid phase. In our case of the magnetic fiber suspension, this fraction was adjusted to a much higher value, $\phi=70 \%$, which allowed us to explain a high loss modulus observed in experiments. Such big quantity of pivoting chains seems to be too important. However, apart from these chains, bridging structures cross-linking two percolating aggregates can exist and contribute to the viscous response of the suspension. So, the factor $\phi$ would rather stand for the relative quantity of the aggregates admitting non-affine motion, including pivoting, free and bridging 
aggregates. Optical microscopy observations [Fig. 1b] revealed a relatively large quantity of these structures in fiber suspensions, compared to suspensions of spherical particles. This could explain an enhanced viscous response of the fiber suspensions. Of course, the variety of the intricate structures observed in fiber suspensions could generate a large spectrum of relaxation times, not accounted in our theory. However, our single-relaxation time model is the first necessary step to the understanding of the nonlinear viscoelastic response of magnetic fiber suspensions. Note that at large oscillation amplitudes, the motion of pivoting and bridging aggregates can be restricted by the neighboring aggregates, so that they could progressively stick to each other and form thick clusters with a reduced mobility. This could cause irreversible changes of the suspension structure provided that the Brownian motion is absent. Nevertheless, our experiments with increasing and decreasing stress ramps did not reveal a significant hysteresis of the shear moduli. This indicates that the structure can be efficiently reformed by the shear flow, at least in the short time scale, or irreversible transformations might have occurred at longer times. Note finally that the chains of different length are expected to oscillate out-of-phase relative to each other. So, the hydrodynamic screening effects, not accounted in our theory, should be more significant than in the case of a steady shear flow, for which the chains are considered to be more or less parallel to each other [12]. A detailed investigation into these points will be conducted in future.

Klingenberg and coworkers have published several papers on particle-level simulations of oscillatory shear flow of ER fluids [26, 27, 34]. Sim et al. [49] have extended these simulations to the 3D-case. The particle-particle and particle-wall hydrodynamic interactions were rigorously modeled, the electrostatic interactions were considered in the point-dipole limit and the hard-sphere repulsion was introduced to prevent particle overlap. At such conditions, both percolating and pivoting aggregates were observed. Therefore, the rheological behaviors obtained by simulations were essentially similar to the ones predicted by our theory. The exactness brought by particle-level simulation is its unconditional advantage over any semi-rigorous theory. However, both hard sphere repulsion hypothesis and point-dipole approximation could significantly affect the final results. First, the particles in aggregates are always in closed mechanical contact, such that attractive magnetic and repulsive contact forces between them can balance each other. From this point of view, it seems reasonable to consider the aggregates as a continuum (as supposed in our theory) rather than as an ensemble of separated particles, at least at large oscillation amplitudes when the microscopic rearrangements inside aggregates play a minor role. Second, the dipole approximation can underestimate the cohesive strength of aggregates and the critical stress corresponding to their rupture. In our work, we use the Ginder's approach, which accounts more rigorously for magnetic interactions. Finally, it seems to be rather difficult to adapt the particle-level simulations to the elongated particle shape [19], and may be these simulations are not worth the effort, providing that the simple model gives satisfactory predictions of the suspension behavior.

Another interesting approach explaining a high viscous response of ER fluids has been proposed by Martin and co-workers. First, they applied the droplet model to oscillatory shear 
and predicted a $\omega^{1 / 3}$-dependence of the loss modulus, consistent with their experiments [50]. However, the loss modulus was predicted to be much larger than the storage modulus, even at high electric fields, which contradicted to experiments. Furthermore, the energy minimum principle employed in this model may only be applied for low shear states. To overcome this obstacle, Martin and Anderson [51], Martin and Odinek [25], Martin et al. [28] have developed a chain model of ER fluids. They did not suggest gap-spanning structures but rather single straight chains, which length was governed by the balance between magnetic and hydrodynamic forces. They also introduced a phenomenological equation describing a periodic process of chain aggregation and fragmentation. The authors focused on chain dynamics but did not explicitly present any rheological data. De Vicente et al. [32] have applied this model to suspensions of spherical magnetic particles and found the values of the loss modulus to be one order of magnitude lower than those of the storage modulus. We decided to adapt the Martin's model to our case of the magnetic fiber suspension and compare it with our model. We consider free single chains of fibers and use a point-dipole approximation for the magnetic forces acting between fibers. We apply the same Eqs. (3), (9) for the motion of chains and for the stress, with $\phi=1$ and $\Phi_{a}=1$. The main difference between our model and Martin's model is that, in the latter, the aggregates are not gap-spanning even at low shear states and, instead of being of fixed length during an oscillating cycle as in our model, their aspect ratio varies during the oscillation cycle according to the aggregationfragmentation equation, as follows:

$$
\dot{p}=\frac{k}{p}\left[1-\frac{p^{2}}{p_{\max }^{2}}\right]
$$

where $p_{\max }$ is the maximum aspect ratio defined by the balance of magnetic and hydrodynamic forces and $k \propto \mu_{0} H^{2} / \eta_{0}$ is the aggregation rate constant determined to a multiplier $k_{0}: k=k_{0} \mu_{0} H^{2} / \eta_{0}$. Since in all above equations, the aspect ratio is associated to the factor $\Omega \equiv p^{2} / \ln (2 p)$, it is more convenient to write the last equation in terms of this factor. Neglecting the variation of the logarithmic term, the Eq. (15) reads:

$$
\begin{gathered}
\dot{\Omega}=2 k\left[1-\frac{\Omega}{\Omega_{\max }}\right], \\
\Omega_{\max }= \begin{cases}\frac{3 \chi_{f}^{2} \mu_{0} H^{2}}{8(l / a)^{2} \eta_{0} \dot{\gamma}}\left(\frac{1}{\tan \theta}-\frac{2 \tan \theta}{\left[2+\chi_{f}(1-\Phi)\right]^{2}}\right), \quad \theta \dot{\gamma} \geq 0 \text { and } \Omega \leq\left(\frac{b}{2 a \cos \theta}\right)^{2} \\
\left(\frac{b}{2 a \cos \theta}\right)^{2}, \quad \text { otherwise }\end{cases}
\end{gathered}
$$

where $l$ and $a$ are the fiber semi-length and radius, respectively, $b$ is the width of the rheometer gap. The maximum chain aspect ratio (or factor $\Omega$ ) is given by the Eq. (17), which postulates that the chain length is either bounded by the equilibrium length corresponding to 
the equality of the hydrodynamic and magnetic forces (upper expression) or by the width of the rheometer gap, $b$ (lower expression). Note that in their model, Martin and Odinek [25] did not take into account that the chain length may overcome the rheometer gap. So, at $\theta \dot{\gamma}<0$, the longitudinal hydrodynamic force is no more tensile but compressive, and the authors considered the maximum chain length to be infinite at $\theta \dot{\gamma}<0$. We think that such approximation could seriously overestimate the chain length, especially at low frequencies and strains, therefore, we include the limitation of the chain length by the rheometer gap.

The Eqs. (3)-(5), (9), (16), (17) (Eq. (16) replaces Eq. (8)) form a closed system of equations for the modified Martin's model and are solved for the sinusoidal stress input, $\sigma(t)=\sigma_{0} \cos (\omega t)$ and under initial conditions: $\theta(0)=0, \Omega(0)=500$. The free parameter $k_{0}$ is varied in the range of $10^{-3}<k_{0}<10^{3}$. Using this aggregation/fragmentation model, we have calculated the frequency dependence of the fundamental shear moduli at $\sigma_{0}=244 \mathrm{~Pa}$ and found the best fit to the experimental results at $k_{0}=0.02$. In Fig. $4 \mathrm{~b}$, we compare the results of the modified Martin's model (curves denoted by "b") with the results of our model (curves denoted by "a"). We observe that, in the frequency range, $f<1 \mathrm{~Hz}$, the aggregation/fragmentation model fits better the experimental results than our model. In fact, this model allows existence of particle chains with the length higher than the one defined by the equilibrium of hydrodynamic and magnetic forces. Even though these chains have a short lifetime compared to the oscillation period, they contribute significantly to both storage and loss moduli. Furthermore, the suspension viscoelastic response is described by, at least, two time scales: (i) the above considered hydrodynamic relaxation time $\tau$ and (ii) the characteristic time of aggregation/fragmentation, $\tau_{a}=1 / k$. Such two relaxation time model gives a power-law frequency response of the shear moduli at low frequencies: $G_{1}{ }^{\prime} \propto \omega^{2.8}$ and $G_{1}{ }^{\prime} \propto \omega^{0.69}$.

The difference in viscoelastic response obtained by both models [Fig. 4b] arises likely due to different microstructures supposed by these models. In the Martin's model only free particle chains, that may break and grow periodically, are considered, while our model imposes pivoting chains of a fixed length attached by one of its ends to a rheometer wall. In reality, the suspension microstructure is more complex [Fig. 1b] and combines the features of both model structures. In a real cross-linked network, some free branches simulated by our model could exist. On the other hand, under oscillatory shear, different branches of the network may overlap each other and may periodically break and reform. The kinetics of such process could be somewhat similar to the aggregation / fragmentation kinetics described by Martin's model. In perspective, we are planning to develop a network model of the suspension microstructure and introduce an aggregation-fragmentation mechanism taking into account eventual collisions and overlapping of the neighboring branches. Anyway, the results of the present work will be helpful for this future investigation.

\section{Concluding remarks}

In this work, we have studied a LAOS response of a magnetic fiber suspension in the presence of a uniform magnetic field applied perpendicularly to the direction of shear. The experiments 
and simulations were carried out in a controlled stress mode. The main results of this study can be summarized as follows:

1. In experiments, the fundamental shear moduli developed a staircase-like stress dependence with, at least, two viscoelastic quasi-plateaus followed by a gradual decrease with the stress. The first plateau was observed at very small deformations, $10^{-4}<\gamma_{0}<10^{-3}$ and attributed to the linear viscoelastic regime corresponding to short-scale rearrangement of particles inside the aggregates. The second quasi-plateau occurred at larger deformations, $0.1<\gamma_{0}<1$, and was associated with the onset of purely macroscopic deformation of aggregates. An abrupt decrease of the moduli after the second quasi-plateau was explained by a significant increase in amplitudes of oscillations of aggregates as well as by their rupture by the hydrodynamic tensile force exerted by the solvent. Both moduli were of the same order of magnitude at the level of the second viscoelastic plateau, while the storage modulus became much lower than the loss modulus at high oscillation amplitudes. Lastly, a local minimum was observed in the stress dependence of the storage modulus at high stresses, which seems to be reproducible and unexpected behavior.

2. For high enough stress, the experimental frequency dependence of the shear moduli followed power-law behavior at low frequency limit $\left(G_{1}{ }^{\prime} \propto \omega^{1.34}, G_{1}{ }^{\prime} \propto \omega^{0.73}\right)$, while at high frequencies, the storage modulus developed a plateau. The power-law exponents were lower than those typical for a single relaxation time Maxwell fluid $\left(G_{1}{ }^{\prime} \propto \omega^{2}, G_{1}{ }^{\prime} \propto \omega^{1}\right)$, suggesting existence of a relaxation time spectrum. This spectrum could be caused by a polydispersity of the aggregates or by complex cross-linking structure of the fiber suspension.

3. The observed rheological behaviors are tightly bound to the suspension microstructure, which evolves with the growing oscillation amplitude. We suggested five simple microstructures replacing each other with the growing stress. A coexistence of percolating and pivoting aggregates at low stresses allowed us to explain a relatively high viscous response of the suspension. To describe the experimental results, we constructed a theoretical model allowing calculations of macroscopic rheological properties from the behavior of the proposed microstructures. The model contains a single free parameter - the fraction $\phi$ of pivoting chains in the suspension. Involving a single time hydrodynamic relaxation, this model fits the experimental stress dependence of the shear moduli reasonably well and reproduces the shape of the measured strain waveforms and of the stress-strain loops (Lissajous plots). The proposed model also gives a qualitative correspondence for the frequency dependence of the shear moduli, however, the quantitative comparison between theory and experiments is less obvious.

4. The frequency behavior can be better reproduced by a modified Martin's model, in which only free non-interacting particle chains are considered and their periodic aggregation and fragmentation is taken into account. However, in the present form, this model neglects important behaviors of the suspension structure and may be applied safely only for very diluted suspensions. The real structure of the fiber suspension should combine the features 
described by both models - existence of percolating network with free branches from the one hand (our model) and aggregation/fragmentation of structure elements from the other hand (Martin's model). Therefore, a synthesis of both models, together with the consideration of a complex cross-linked network, would significantly improve the theoretical description of the viscoelastic response of magnetic suspensions. Here, the polymer dynamics theories [52] could serve as a solid base.

In our study, we did not account for solid friction between fibers in aggregates. Friction between fibers could play an important role in their microscopic rearrangement at small amplitude oscillatory shear and could significantly enhance the suspension shear moduli at the first viscoelastic plateau. In particular, Lopez-Lopez et al. [53] have recently explained high values of MR fluid loss modulus by a solid friction between particles at their microscopic displacements relative to each other. However, at large amplitudes, microscopic motions of particles and, consequently, interparticle friction are expected to play a non-negligible role but hydrodynamic and magnetic interactions are expected to be predominant. In order to estimate correctly the contribution of friction into the shear moduli, we must be able to determine the friction coefficient. So, the measurements of this quantity are required for the future progress in this direction.

\section{Acknowledgements}

Biomag (PACA), Eureka E! 3733 Hydrosmart project, Dynxperts project (ref. FP7-2010NMP-ICT-FoF-260073), FIS2009-07321 (MICINN, Spain), P08-FQM-3993, P09-FQM-4787 (Junta de Andalucía, Spain) and Cooperation Program between CNRS and BRFFR $\left(\mathrm{N}^{\circ}\right.$ 23178, France-Belarus) are acknowledged for the financial support. One of the authors (M.T.L.-L.) also acknowledges financial support by the University of Granada (Spain).

\section{Appendix. Hydrodynamic torque acting on pivoting chains}

Consider a long chain attached by its lower end to the wall and having a free upper end, as depicted in Fig. 1h. This chain is subject to a simple shear flow, such that the velocity profile of the suspending fluid can be presented as the sum of a constant velocity $v_{c}$ in the chain center of mass $\mathrm{C}$ and a linear velocity profile:

$$
v=v_{c}+\dot{\gamma} z \cos \theta=\dot{\gamma} L \cos \theta+\dot{\gamma} z \cos \theta,
$$

with $z$ - longitudinal coordinate along the chain major axis (the origin is placed into the center of mass $\mathrm{C}$ of the chain). The constant velocity $v_{c}$ creates, inter alia, a hydrodynamic drag force $F_{\perp}$ perpendicular to the aggregate major axis. This force creates, in its term, a torque $F_{\perp} L$, which tends to tilt the aggregate in the direction of shear. From the other hand, the linear velocity profile also exert a torque on the chain, $T_{h C}$. So, the resultant torque is

$$
T_{h}=F_{\perp} L+T_{h C}
$$


The expressions for the drag force $F_{\perp}$ and the torque $T_{h C}$ are given by the slender body theory [37]:

$$
\begin{aligned}
& F_{\perp}=8 \pi \eta_{0} U L \varepsilon \frac{1+0.307 \varepsilon}{1+0.5 \varepsilon}, \\
& T_{h C}=\frac{8}{3} \pi \eta_{0} \Omega L^{3} \varepsilon \frac{1+0.64 \varepsilon}{1-0.5 \varepsilon}
\end{aligned}
$$

where $U=\left(\dot{\gamma} \cos ^{2} \theta-\dot{\theta}\right) L$ is the normal to the chain component of the solvent velocity in the coordinate frame of the chain; $\Omega=\dot{\gamma} \cos ^{2} \theta-\dot{\theta}$ is the solvent angular velocity in the coordinate frame of the chain, $\varepsilon=1 / \ln (2 p)$. Substituting the Eqs. (A.3) and (A.4) into Eq.(A.2), we obtain the final expression for the hydrodynamic torque acting on pivoting chains (left-hand side of the Eq. (1)) with a numerical factor $f^{\perp}=(1+0.64 \varepsilon) /(1-0.5 \varepsilon)+3 \cdot(1+0.307 \varepsilon) /(1+0.5 \varepsilon)$.

In contrast to pivoting chains, totally free chains [Fig. 1e,f] experience a zero hydrodynamic drug force $F_{\perp}$ and are subjected only to the torque $T_{h C}$. So, the hydrodynamic torque acting on free chains is defined by the same expression (left-hand side of the Eq. (1)), in which the numerical factor $f^{\perp}$ is equal to $f^{\perp}=(1+0.64 \varepsilon) /(1-0.5 \varepsilon)$.

\section{References}

[1] Z. P. Shulman, W. I. Kordonsky, Magnetorheological effect, Nauka i Tehnika, Minsk, 1982 (in Russian)

[2] J. M. Ginder, Behavior of magnetorheological fluids, MRS Bull., 23 (1998) 26-29.

[3] H. Urreta, Z. Leicht, A. Sanchez, A. Agirre, P. Kuzhir, G. Magnac, Hydrodynamic Bearing Lubricated With Magnetic Fluids, J. Intel. Mater. Syst. and Struct. DOI: $10.1177 / 1045389$ X09356007

[4] G. Ngatu, N.M. Wereley, J. Karli, R.C. Bell, Dimorphic Magnetorheological Fluids: Exploiting Partial Substitution of Microspheres by Nanowires, Smart Materials and Structures. 17 (2008) 045022.

[5] R.C. Bell, E.D. Miller, J.O. Karli, A.N. Vavreck, D.T. Zimmerman, Influence of particle shape on the properties of magnetorheological fluids, Int. J. Mod. Phys. B, 21 (2007) 50185025 .

[6] R.C. Bell, J.O. Karli, A.N. Vavreck, D.T. Zimmerman, G.T. Ngatu, N.M. Wereley, Magnetorheology of submicron diameter iron microwires dispersed in silicon oil, Smart Mater. Struct., 17 (2008) 015028.

[7] M. T. López-López, G. Vertelov, P. Kuzhir, G. Bossis, J.D.G. Durán, New magnetorheological fluids based on magnetic fibers, J. Mater. Chem., 17 (2007) 3839-3844. 
[8] M. T. López-López, P. Kuzhir, G. Bossis, Magnetorheology of fiber suspensions. I. Experimental, J. Rheol., 53 (2009) 115-126.

[9] J. de Vicente, J.P. Segovia-Guitérrez, E. Anablo-Reyes, F. Vereda, R. Hidalgo-Alvarez, Dynamic rheology of sphere- an rod-based magnetorheological fluids, J.Chem. Phys. 131 (2009) 194902.

[10] A. Gómez-Ramírez, M.T. López-López, J.D.G. Durán, F. González-Caballero, Influence of particle shape on the magnetic and magnetorheological properties of nanoparticle suspensions, Soft Matter, 5 (2009) 3888-3895.

[11] P. Kuzhir, M. T. López-López, G. Bossis, Magnetorheology of fiber suspensions. II. Theory, J. Rheol., 53 (2009) 127-151.

[12] A. Gómez-Ramírez, P. Kuzhir, M.T. López-López, G. Bossis, A. Meunier, J.D.G. Durán, Steady shear flow of magnetic fiber suspensions: theory and comparison with experiments, J.Rheol., 55 (2011) 43-67.

[13] K. Asano, H. Suto, K. Yatsuzuka, Influence of the particle configuration on electrorheological effect, J. Electrostat. 40-41 (1997) 573-578.

[14] Y.Otsubo, Electrorheology of whiskers suspensions, Colloids and surfaces A 153 (1999) 459-466.

[15] K. Tsuda, Ya. Takeda, H. Ogura, Ya. Otsubo, Electrorheological behavior of whisker suspensions under oscillatory shear, Colloids and Surfaces A 299 (2007) 262-267.

[16] M.M. Ramos-Tejada, M.J. Espin, R. Perea, A.V. Delgado, Electrorheology of suspensions of elongated goethite particles, J. NonNewt. Fluid Mech. 159 (2009) 34-40.

[17] R.C. Kanu, M.T. Shaw, Enhanced electrorheological fluids using anisotropic particles, J. Rheol. 42 (1998) 657-660,.

[18] A. Kawai, U. Kunio, I. Fumikazu, Effects of Shape and Size of Dispersoid on Electrorheology, Int. J. Mod. Phys. B 16, (2002) 2548-2554.

[19] Ya. K. Kor, H. See, The electrorheological response of elongated particles, Rheol. Acta 49 (2010) 741-756.

[20] A.J. Giacomin, J.M. Dealy, in: “Techniques in Rheological Measurements”, Vol.4, A.A. Collyer, Ed., Chapman and Hall, London 1993, chapter 9.

[21] K. Hyun, J. G. Nam, M. Wilhelm, K. H. Ahn, S. J. Lee, Nonlinear response of complex fluids under LAOS (large amplitude oscillatory shear) flow, Korea-Australia Rheology Journal 15 (2003) 97-105.

[22] M. Wilhelm, Fourier-Transform Rheology, Macromol. Mater.Eng. 287 (2002) 83-105. 
[23] R. H. Ewoldt, A. E. Hosoi, G. H. McKinley, New measures for characterizing nonlinear viscoelasticity in large amplitude oscillatory shear, J. Rheol. 52 (2008) 1427-1458.

[24] D.R. Gamota, A.S. Wineman, F.E. Filisko, Fourier transform analysis: nonlinear dynamic response of an electrorheological material, J. Rheol. 37 (1993) 919-933.

[25] J.E. Martin, J. Odinek, Aggregation, Fragmentation, and the Nonlinear Dynamics of Electrorheological Fluids in Oscillatory Shear, Phys. Rev. Lett. 75 (1995) 2827-2830.

[26] M. Parthasarathy, D.J. Klingenberg, A microstructural investigation of the nonlinear response of electrorheological suspensions, Rheol. Acta 34 (1995) 417-429.

[27] M. Parthasarathy, D.J. Klingenberg, Large amplitude oscillatory shear of ER suspensions, J. NonNewt. Fluid Mech. 81 (1999) 83-104.

[28] J.E. Martin, J. Odinek, Th.C. Halsey, R. Kamien, Structure and dynamics of electrorheological fluids, Phys. Rev. E, 57 (1998) 756-775.

[29] W.H. Li, H. Du, G. Chen, S.H. Yeo, N. Guo, Nonlinear viscoelastic properties of MR fluids under large-amplitude-oscillatory-shear, Rheol. Acta 42 (2003) 280-286.

[30] W.H. Li, H. Du, N. Guo, Dynamic behavior of MR suspensions at moderate flux densities, Mater. Sci. Eng. A 371 (2004) 9-15.

[31] J. Claracq, J. Sarrazin, J.-P. Montfort, Viscoelastic properties of magnetorheological fluids, Rheol. Acta 43 (2004) 38-49.

[32] J. de Vicente, M.T. López-López, J.D.G. Durán, G. Bossis, A slender-body micromechanical model for viscoelasticity of magnetic colloids: Comparison with preliminary experimental data, J. Coll. Int. Sci. 282 (2005) 193-201.

[33] T.C.B. McLeish, T Jordan, M.T. Shaw, Viscoelastic response of electrorheological fluids. I. Frequency dependence, J. Rheol. 35 (1991) 427-448.

[34] D.J. Klingenberg, Simulation of the dynamic oscillatory response of electrorheological suspensions: Demonstration of a relaxation mechanism, J. Rheol. 37 (1993) 199-214.

[35] T. Jordan, M.T. Shaw, T.C.B. McLeish, Viscoelastic response of electrorheological fluids. II. Field strength and strain dependence, J. Rheol., 36 (1992) 441-463.

[36] D. Jiles, Introduction to Magnetism and Magnetic Materials, Chapman \& Hill, London, 1991.

[37] G.K. Batchelor, Slender-body theory for particles of arbitrary cross-section in Stokes flow, J. Fluid. Mech. 44 (1970) 419-440.

[38] G.K. Batchelor, The stress generated in a non-dilute suspension of elongated particles by pure straining motion, J. Fluid. Mech. 46 (1971) 813-829. 
[39] H.M. Laun, C. Gabriel, C. Kieburg, Effect of wall materials and roughness on transmittable shear stress of magnetorheological fluids, submitted (2010)

[40] M.T. López-López, P. Kuzhir, J.D.G. Durán, G. Bossis, Normal stresses in a shear flow of magnetorheological suspensions: Viscoelastic versus Maxwell stresses, J.Rheol. 54 (2010) 1119-1136.

[41] Berthier, S., “Optique des milieux composites”, Polytechnica, Paris (1993).

[42] J. M. Ginder, L. C. Davis, L. D. Elie, Rheology of magnetorheological fluids: Models and measurements, Int. J. Mod. Phys. B 10 (1996) 3293-3303.

[43] J. Läuger, H. Stettin, Differences between stress and strain control in the non-linear behavior of complex fluids, Rheol. Acta 49 (2010) 909-930.

[44] Ch.W. Macosco, Rheology. Principles, Measurements, and Applications, Wiley-VCH, Inc., New York, 1994, pp. 217-222.

[45] G. Bossis, E. Lemaire, O. Volkova, H. Clercx, Yield stress in magnetorheological and electrorheological fluids: A comparison between microscopic and macroscopic structural models, J. Rheol. 41 (1997) 687-704.

[46] H. Brenner, Rheology of a dilute suspension of axisymmetric Brownian particles, Int. J. Multiphase Flow 1 (1974) 195-341.

[47] V.N. Pokrovskiy, Statistical mechanics of diluted suspensions, Nauka, Moscow, 1978 (in Russian).

[48] R.B. Bird, R.C. Armstrong, O. Hassager, Dynamics of Polymeric Liquids: Voulme I. Fluid Mechanics, Wiley, New York, 1987.

[49] H. G. Sim, K.H. Ahn, S.J. Lee, Three-dimensional dynamics simulation of electrorheological fluids under large amplitude oscillatory shear flow, J. Rheol. 47 (2003) 879-895.

[50] J. E. Martin, D. Adolf, Th. C. Halsey, Electrorheology of a Model Colloidal Fluid, J.Coll. Int. Sci., 167 (1994) 437-452.

[51] J.E. Martin, R.A. Anderson, Chain model of electrorheology, J.Chem.Phys 104 (1996) 4814-4827.

[52] R.B. Bird, O. Hassager, R.C. Armstrong, Ch. F. Curtiss, Dynamics of Polymeric Liquids Volume II. Kinetic Theory, Wiley Interscience, New York, 1987.

[53] M.T. Lopez-Lopez, L. Rodriguez-Arco, A. Zubarev, L. Iskakova, J.D.G. Duran, Effect of gap thickness on the viscoelasticity of magnetorheological fluids, J.Appl.Phys., 108 (2010) doi:10.1063/1.3498804 